Check for updates

Cite this: RSC Adv., 2018, 8, 31471

\title{
Synthesis of zeolitic imidazolate framework-8 on polyester fiber for $\mathrm{PM}_{2.5}$ removal
}

\author{
Yongyong Zhang, Ying Jia and Li'an Hou (D)*
}

Zeolitic imidazolate framework-8 (ZIF-8) was introduced on the surface of fibers of a non-woven fabric made of polyethylene terephthalate (PET). The phase structure, morphology, textural properties and chemical structure were studied using X-ray diffraction (XRD), scanning electron microscopy (SEM), nitrogen $\left(\mathrm{N}_{2}\right)$ adsorption analyzer and Fourier transform infrared spectroscopy (FTIR). The XRD, SEM and FTIR results indicated that ZIF- 8 had been successfully coated on the PET fibers. $\mathrm{N}_{2}$ adsorption analyses suggested that addition of ZIF- 8 significantly increased the specific surface and micropore volume of composites. The $\mathrm{PM}_{2.5}$ removal experiment showed the filtration efficiency of ZIF-8/PET was twice as high as that of pure PET. Meanwhile, ZIF-8/PET also displayed a low pressure drop and good recycling performance. This research provided a facile method to deposit active ZIF- 8 onto polymers with low air resistance, and that ZIF-8/PET was a good candidate as an air filter for $\mathrm{PM}_{2.5}$ removal.

Received 30th July 2018

Accepted 1st September 2018

DOI: $10.1039 / \mathrm{c} 8 \mathrm{ra0} 6414 \mathrm{~h}$

rsc.li/rsc-advances

As a subclass of metal-organic frameworks (MOFs), zeolitic

\section{Introduction}

The World Health Organization (WHO) estimated that total global mortality due to air pollution was 7 million in $2012 .^{1}$ Particulate matter (PM), especially $\mathrm{PM}_{2.5}$ (particulates with a size of $2.5 \mu \mathrm{m}$ or less), has become one of the largest contributors to air pollution. Over the last decade, PM has increased in prominence globally and a large volume of epidemiological and toxicological evidence has indicated that adverse health effects in both developing and developed countries can be linked to this increase. ${ }^{2-4}$ Health effects related to PM include increased acute and chronic respiratory and cardiovascular morbidity, and even mortality in both children and adults. ${ }^{5,6}$ Besides, PM also has adverse effects on visibility and climate change. ${ }^{7,8}$ Many researchers have developed air filters for application in air purification. ${ }^{9}$ For example, Wang et al. ${ }^{\mathbf{1 0}}$ prepared large scale ultrafine chitosan hybrid nanofibers containing $\mathrm{TiO}_{2}$ and/or Ag nanoparticles using a needleless electrospinning method for air filtration. Jeong et al. ${ }^{\mathbf{1 1}}$ presented an environmental application demonstration of a $\mathrm{Ag}$ nanowire percolation network for a novel, electrical type transparent, reusable, and active $\mathrm{PM}_{2.5}$ air filter. Kim et al. ${ }^{\mathbf{1 2}}$ reported a surface-modified polymer nanofiber membrane for high-efficiency microdust capturing. As for the use to purify air, low air-resistance should be concerned to meet the enough ventilation rate and low noise. Non-woven fabrics are usually used as the coarse filter to remove suspended particulate matters. Here, a cheap non-woven fabric made of polyethylene terephthalate (PET) was selected. imidazolate frameworks (ZIFs) are porous crystals with zeolitetype structures built by metal ions and imidazolate ligands. ${ }^{13}$ ZIFs possess the unique properties of high crystallinity, large surface area, exceptional chemical and thermal stability and functional tenability. As a result, ZIFs have gained considerable attention for their potential application in gas storage, $\mathbf{C O}_{2}$ capture, separation, sensing, catalysis, drug delivery and water treatment. ${ }^{14-21}$ Zeolitic imidazolate framework-8 (ZIF-8), with sodalite (SOD) topology constructed from $\mathrm{Zn}$ and Hmim is one of the most investigated ZIF materials. ${ }^{22,23}$ Recently, ZIF-8 had been used for $\mathrm{PM}_{2.5}$ removal experiment. ${ }^{24}$ The result showed that ZIF-8 possessed excellent $\mathrm{PM}_{2.5}$ capture property. Therefore, immobilization of ZIF-8 on PET will be a promising method to improve filtration performance.

Herein, ZIF-8 crystals were synthesized by mixing 2-methylimidazole with zinc nitrate hexahydrate in methanol solution. ZIF-8/PET was fabricated by growing ZIF-8 on the 3aminopropyltriethoxysilane-modified PET in one step. X-ray diffraction (XRD), scanning electron microscopy (SEM) and gas sorption instrument as well as Fourier transform infrared (FTIR) spectra were employed for characterizing the structure of ZIF-8 and ZIF-8/PET. Furthermore, the $\mathrm{PM}_{2.5}$ removal property of ZIF-8/PET was also investigated.

\section{Experimental}

\subsection{Materials}

Zinc nitrate hexahydrate (>99.0\%), 2-methylimidazole (>98.0\%) and methanol (>99.5\%) were purchased from Sigma Aldrich. 3Aminopropyltriethoxysilane (APTES, >99\%) and sodium 
formate (>99.0\%) were obtained from Aladdin, Shanghai China. PET materials purchased from Beijing SIBAOTE Company (China) with thickness of $5 \mathrm{~mm}$ were used as substrate for the deposition of ZIF-8. All chemicals were of analytical grade and used without any purification. Deionized water was used throughout this work.

\subsection{Synthesis of ZIF-8}

Typically, $2.932 \mathrm{~g}$ zinc nitrate hexahydrate was first dissolved in $50 \mathrm{~mL}$ methanol, which was labeled as solution A. $1.214 \mathrm{~g} \mathrm{2-}$ methylimidazole was dissolved in $50 \mathrm{~mL}$ methanol, which was labeled as solution B. The solution B was slowly poured into solution A under stirring at room temperature $\left(25 \pm 3{ }^{\circ} \mathrm{C}\right)$. The mixture solution was stirred for $10 \mathrm{~min}$ at constant speed of $200 \mathrm{rpm}$. Then the mixture solution was poured into a Teflonlined stainless steel autoclave and heated to $120{ }^{\circ} \mathrm{C}$ in an air oven for $4 \mathrm{~h}$. The final product were washed with methanol for 3 times and then dried in air for subsequent characterization.

\subsection{Synthesis of ZIF-8 on polyester fibers}

First polyester fibers were washed with deionized water and methanol to remove residuals on the surface and dried under an ambient atmosphere. Then fibers were modified with APTES ( $2 \mathrm{wt} \%$ in methanol) at $25{ }^{\circ} \mathrm{C}$ for $1 \mathrm{~h}$, leading to APTES layers deposited on the support surface.

$2.932 \mathrm{~g}$ zinc nitrate hexahydrate was first dissolved in $50 \mathrm{~mL}$ methanol, which was labeled as solution A. $1.214 \mathrm{~g}$ 2methylimidazole and $0.671 \mathrm{~g}$ sodium formate was dissolved in $50 \mathrm{~mL}$ methanol, which was labeled as solution B. The solution B was slowly poured into solution A under stirring at room temperature $\left(25 \pm 3{ }^{\circ} \mathrm{C}\right)$. The mixture solution was stirred for $10 \mathrm{~min}$ at constant speed of $200 \mathrm{rpm}$. Then the APTES modified fibers were vertically placed in a Teflon-lined stainless steel autoclave which was filled with the mixture solution and heated to $120^{\circ} \mathrm{C}$ in an air oven for $4 \mathrm{~h}$. The final product were washed with methanol for 3 times to remove any possible residuals and then dried in air for subsequent characterization.

\subsection{Characterization}

X-ray diffraction (XRD, X'PERT MRD, PANalytic, Holland) was performed using $\mathrm{Cu} \mathrm{K} \alpha$ radiation at $40 \mathrm{kV}$ and $50 \mathrm{~mA}$ to determine the crystalline. The $2 \theta$ scanning range was $5-35^{\circ}$, the step size was $0.008^{\circ}(2 \theta)$ and the scanning speed was $3^{\circ}(2 \theta) \min ^{-1}$. Scanning electron microscopy (SEM, TESCAN VEGAII XMUINCN, Czech Republic) in combination with energy dispersive spectrometer (EDS, OXFORD INCA) were used to characterize the morphology and particles size. The accelerating voltage and current of electron beam are $21 \mathrm{keV}$ and $0.2 \mathrm{nA}$ respectively. Each sample was coated with a very thin gold using a sputter coater under certain high-vacuum conditions to achieve conductivity and vacuum durability and then put into the SEM chamber. The surface area and adsorption-desorption isotherm measurements were carried out on a Quantachrome Autosorb-iQ gas sorption instrument at $77 \mathrm{~K}$ using liquid nitrogen as coolant. The sample was degassed at $150{ }^{\circ} \mathrm{C}$ for $3 \mathrm{~h}$ under vacuum before the measurements. The textural properties were determined via nitrogen sorption at $-196{ }^{\circ} \mathrm{C}$. Fourier transform infrared (FTIR) spectra in the range $4000-400 \mathrm{~cm}^{-1}$ were obtained on a Thermo Fisher Nicolet 6700 FTIR spectrometer using the $\mathrm{KBr}$ wafer technique. Pictures of the characterization setups were shown in Fig. 1 and 2.

\section{5 $\mathbf{P M}_{2.5}$ removal experiment}

The $\mathrm{PM}_{2.5}$ was generated from incense smoke by burning. The inflow concentration was controlled by diluting the smoke by air to a hazardous pollution level equivalent to the $\mathrm{PM}_{2.5}$ mass concentration $\sim 600 \mu \mathrm{g} \mathrm{m}^{-3}$. The wind velocity used in the test was $0.2 \mathrm{~m} \mathrm{~s}^{-1}$ and the relative humidity was $50 \pm 5 \% . \mathrm{PM}_{0.5}$, $\mathrm{PM}_{1.0}$ and $\mathrm{PM}_{2.5}$ concentration were detected by DustTrak 8530 aerosol monitor (TSI Inc., USA) and the removal efficiency was calculated by comparing the mass concentration before and after filtration. The size distribution of the original $\mathrm{PM}_{2.5}$ source was presented in Table 1 . The pressure drop was measured by a pressure gauge (DP1000-IIIC, Shanghai, China). Fifty groups of mass concentration data were collected to give the average

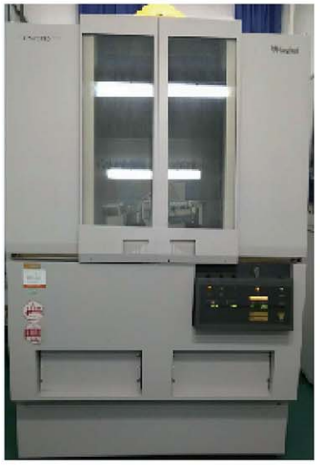

X-ray diffraction

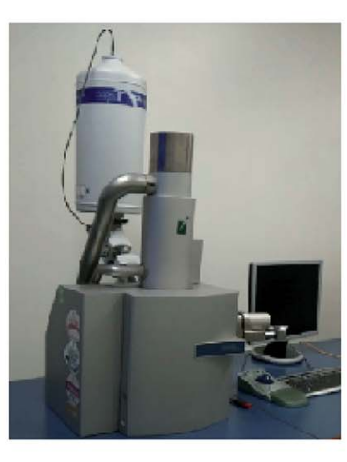

Scanning electron microscopy

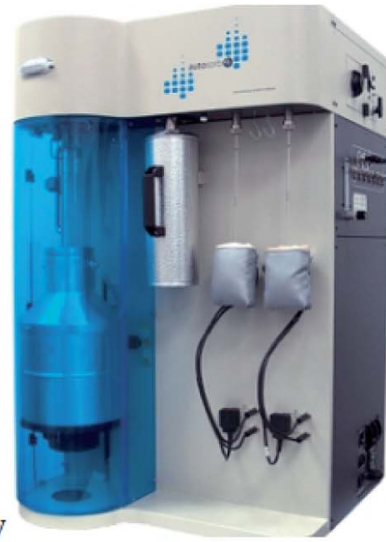

Gas sorption instrument

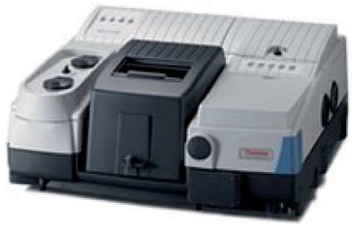

Fourier transform infrared spectrometer

Fig. 1 Pictures of the characterization setups. 


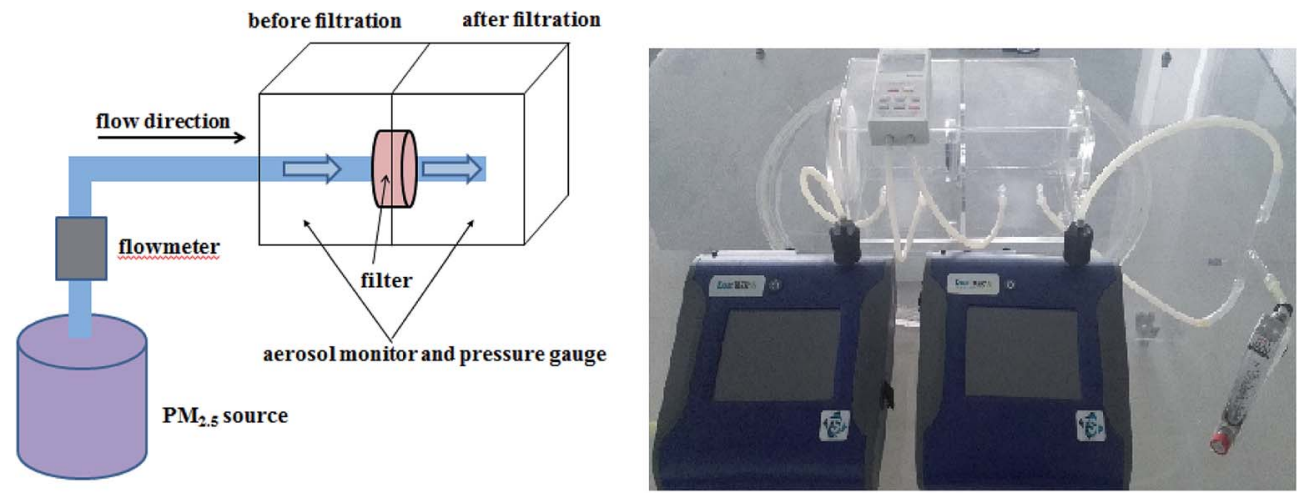

Fig. 2 Schematic diagram and photo-image of the experimental setup for $\mathrm{PM}_{2.5}$ removal experiment.

Table 1 The size distribution of the original $\mathrm{PM}_{2.5}$ source

\begin{tabular}{llll}
\hline Particle size interval & $0-0.5 \mu \mathrm{m}$ & $0-1.0 \mu \mathrm{m}$ & $0-2.5 \mu \mathrm{m}$ \\
Mass percent (\%) & 68.12 & 85.48 & 100
\end{tabular}

concentration. The removal efficiency was calculated by the following equation:

$$
\text { Removal efficiency }=\left(1-c / c_{0}\right) \times 100 \%
$$

where $c$ and $c_{0}$ are the average $\mathrm{PM}_{2.5}$ concentrations after and before passing through the filter.

\section{Results and discussion}

\subsection{XRD analysis}

The phase structure of different samples was determined by XRD. The characteristic diffraction peaks at $2 \theta=7.4^{\circ}, 10.4^{\circ}$, $12.7^{\circ}, 14.7^{\circ}, 16.4^{\circ}, 18.0^{\circ}, 22.1^{\circ}, 24.5^{\circ}, 26.7^{\circ}$ and $29.6^{\circ}$ for ZIF-8 sample were observed clearly in Fig. 3, which can be assigned to (011), (002), (112), (022), (013), (222), (114), (233), (134) and (044) planes respectively. The prominent reflections agreed well

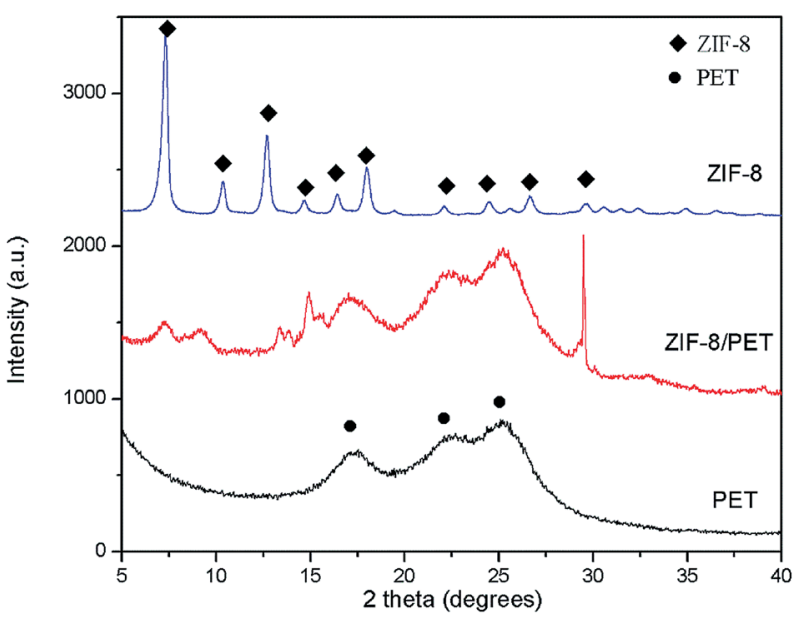

Fig. 3 XRD patterns of ZIF-8, PET and ZIF-8/PET. with previous reports, confirming the typical sodalite structure of ZIF-8. ${ }^{13,25,26}$ Three diffraction peaks located around $17.1^{\circ}$, $22.6^{\circ}$ and $26.2^{\circ}$ for PET sample were observed, which corresponded to (010), (011) and (100) planes of PET, respectively. ${ }^{27}$ Similar diffraction pattern were shown in ZIF-8/PET sample, revealing that the addition of ZIF-8 did not affect the crystalline form of PET. Furthermore, ZIF-8/PET sample exhibits new peaks at $2 \theta=7.4^{\circ}, 14.7^{\circ}$ and $29.6^{\circ}$, which belongs to ZIF-8, suggesting the presence of ZIF-8 on the surface of PET. Owing to the preferred orientation of crystal growth on PET, the relative peak intensity of ZIF-8/PET is different from that of pure ZIF-8, especially the (011) and (044) plane $\left(2 \theta=7.4^{\circ}\right.$ and $\left.29.6^{\circ}\right) \cdot{ }^{28}$ However, some diffraction peaks positions were slightly shifted towards lower $2 \theta$ angles, indicating a slight expansion of ZIF-8 lattice. This phenomenon may be due to ZIF-8 framework distortion caused by the repulsive interactions between PET and imidazole groups in ZIF-8. Absence of some ZIF-8 diffraction peaks (e.g. $\left.12.7^{\circ}\right)$ in ZIF-8/PET sample could be attributed to the affinity interactions between these lattice planes and APTES or PET.

\subsection{SEM analysis}

Fig. 4 displayed the morphology of synthesized samples. It can be clearly seen from Fig. 4(a) that the diameter of PET fibers was $\sim 20 \mu \mathrm{m}$ and the surface of the raw fibers was smooth with a small amount of impurities. Pure ZIF-8 samples had rhombic dodecahedron morphologies with average particle size of $1 \mu \mathrm{m}$ (Fig. 4(d)), which matched well the results in literature, ${ }^{29}$ confirming that the synthesized method was right. Fig. 4(b and c) is the representative SEM images of ZIF-8/PET with different magnifications. As shown in Fig. 4(b), the surface of the composites was rougher than that of the pure PET due to the adhesion of ZIF-8 crystals to the fiber. ZIF-8 particles may strengthen the interaction between $\mathrm{PM}_{2.5}$ and composites by the open metal sites, functional groups and electrostatic interactions. ${ }^{24}$ Besides, the rough fiber surface may be helpful for the capture of $\mathrm{PM}_{2.5}$. No visible cracks, pinholes or other defects were observed in the surface of ZIF-8/PET, indicating that the contact between ZIF-8 particles and PET was good. These particles had rhombic dodecahedron shape with particle size ranging from $1 \mu \mathrm{m}$ to $2 \mu \mathrm{m}$ (Fig. 4(c)). 


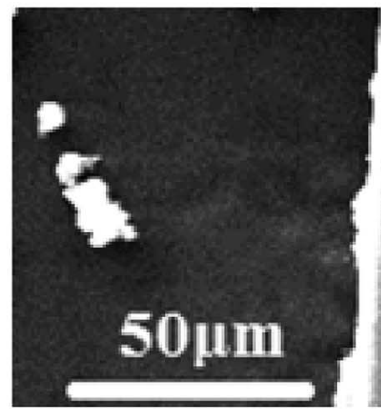

(a)

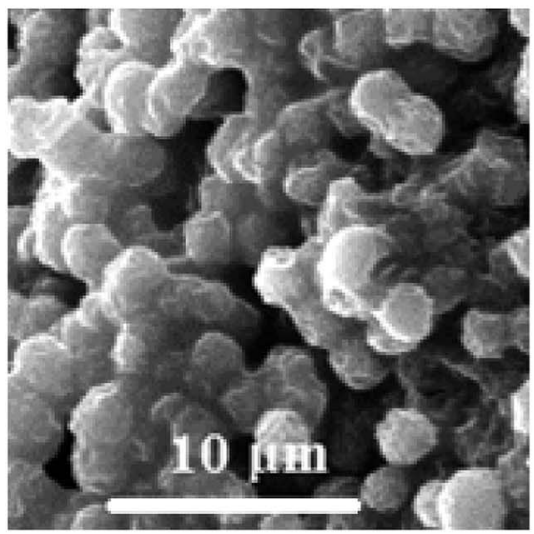

(c)
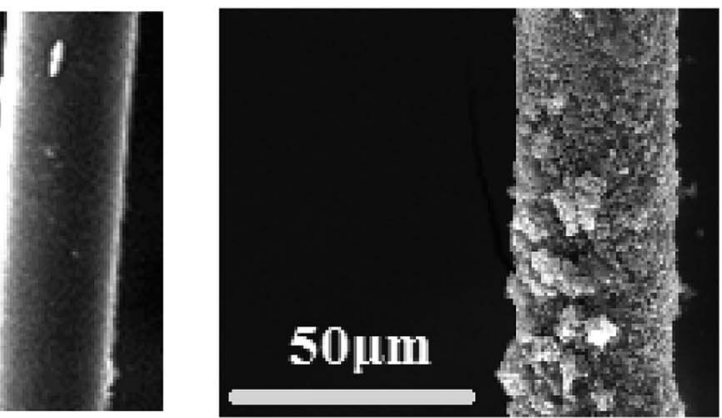

(b)

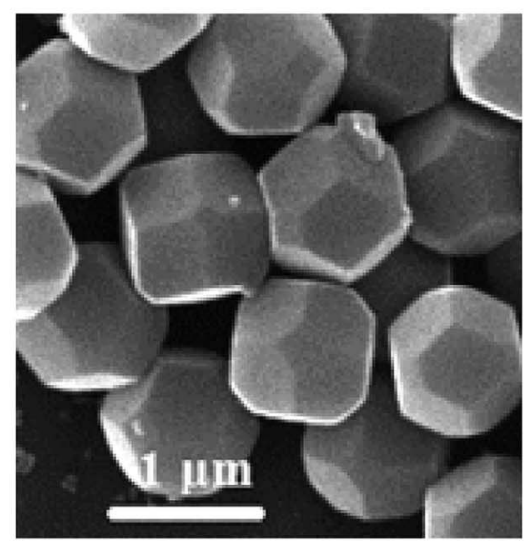

(d)

Fig. 4 SEM images of PET (a), ZIF-8/PET (b and c) and ZIF-8 (d).

\subsection{Textural analysis}

In order to determine the presence of ZIF-8 grains in the PET, nitrogen adsorption-desorption isotherms of ZIF-8, PET and ZIF-8/PET were measured. As shown in Fig. 5(a), the nitrogen adsorption-desorption isotherms of synthesized ZIF-8 displayed typically reversible type I isotherms. The adsorbed nitrogen amount of all the samples at low pressures $\left(P / P_{0}<0.08\right)$ increased steeply, suggesting the existence of micropores. The results were in line with those reported previous. ${ }^{25,30,31}$ Furthermore, the isotherms of ZIF-8 exhibited hysteresis loop near $P / P_{0}=1$, indicating the presence of interparticle mesoporosity and macroporosity between ZIF-8 particles. $^{32}$ The main filtration mechanism is the strong adsorption force of various porous structures on the substance rather than a simple sieve effect. ${ }^{33}$ Indeed, the semi-log plot of nitrogen adsorption isotherms of ZIF-8 showed two steps occurring at $5 \times 10^{-2}$ and 5 $\times 10^{-3} P / P_{0}$ (Fig. 5(b)). The first step could be attributed to a reorganization of the nitrogen molecules caused by strong electrostatic interactions with ZIF framework, while the second step to the gas-induced rotation of the imidazolate linkers upon pressure, i.e. to the gate-opening effect. ${ }^{34}$ The increase in uptake going from the first to the second step was $28.6 \%$, suggesting
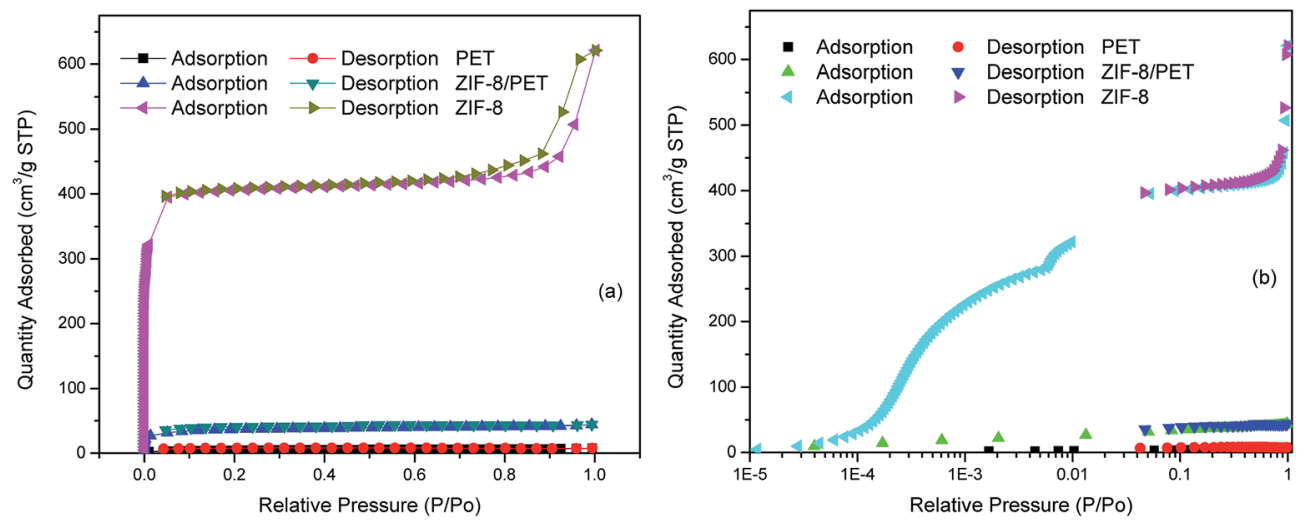

Fig. 5 (a) Nitrogen adsorption-desorption isotherms of ZIF-8, PET and ZIF-8/PET, (b) logarithmic-scale plot of nitrogen adsorption. 
Table 2 Porosity properties of ZIF-8, PET and ZIF-8/PET

\begin{tabular}{lcrc}
\hline Samples & BET surface area $\left(\mathrm{m}^{2} \mathrm{~g}^{-1}\right)$ & Langmuir surface area $\left(\mathrm{m}^{2} \mathrm{~g}^{-1}\right)$ & Micropore volume $^{a}\left(\mathrm{~cm}^{3} \mathrm{~g}^{-1}\right)$ \\
\hline ZIF-8 & 1228 & 1896 & 0.5850 \\
ZIF-8/PET & 116 & 185 & 0.0394 \\
PET & 16 & 31 & 0.0006 \\
${ }^{a} t$-plot method. & & &
\end{tabular}

synthesized ZIF-8 possessed excellent flexibility. The PET and ZIF-8/PET samples also exhibited type I isotherms, which confirmed the presence of micropore structure. Both PET and ZIF-8/PET had relatively little pore structure, then the semi-log plot of nitrogen adsorption isotherms were not discussed here.

Table 2 presented the Brunauer-Emmett-Teller (BET) and Langmuir surface area, and micropore volume calculated with $t$ plot method. The BET, Langmuir surface area and micropore volume increased from $16 \mathrm{~m}^{2} \mathrm{~g}^{-1}, 31 \mathrm{~m}^{2} \mathrm{~g}^{-1}$ and $0.0006 \mathrm{~cm}^{3} \mathrm{~g}^{-1}$ for pure PET to $116 \mathrm{~m}^{2} \mathrm{~g}^{-1}, 185 \mathrm{~m}^{2} \mathrm{~g}^{-1}$ and $0.0394 \mathrm{~cm}^{3} \mathrm{~g}^{-1}$ for ZIF-8/PET, respectively. The additions of ZIF-8 to the PET significantly increased the specific surface and micropore volume, which was helpful for the capture of $\mathrm{PM}_{2.5}$.

\subsection{FTIR spectroscopy analysis}

The chemical structure was defined by using FITR and presented in Fig. 6. It presented remarkable bands at 3455, 3135, 2929, 1635, 1585, 1458, 1425, 1385, 1309, 1146, 995, 760, 694 and $426 \mathrm{~cm}^{-1}$ for ZIF-8 sample. These FT-IR bands were consistent with those previously reported by Cravillon et al., Ordonez et al. and Jomekian et al. ${ }^{35-37}$ The band at $3455 \mathrm{~cm}^{-1}$ might be attributed to the $\mathrm{N}-\mathrm{H}$ stretching vibration of the residual $\mathrm{Hmim}$ and the $\mathrm{O}-\mathrm{H}$ stretching vibration of water from $\mathrm{KBr}$ deliquescence. The peaks at 3135 and $2929 \mathrm{~cm}^{-1}$ were associated with the aromatic and aliphatic $\mathrm{C}-\mathrm{H}$ asymmetric stretching vibrations, respectively. Another signal around $1635 \mathrm{~cm}^{-1}$ arose from the $\mathrm{C}=\mathrm{C}$ stretch mode, while band at $1585 \mathrm{~cm}^{-1}$ corresponded to the $\mathrm{C}=\mathrm{N}$ stretch vibration. The

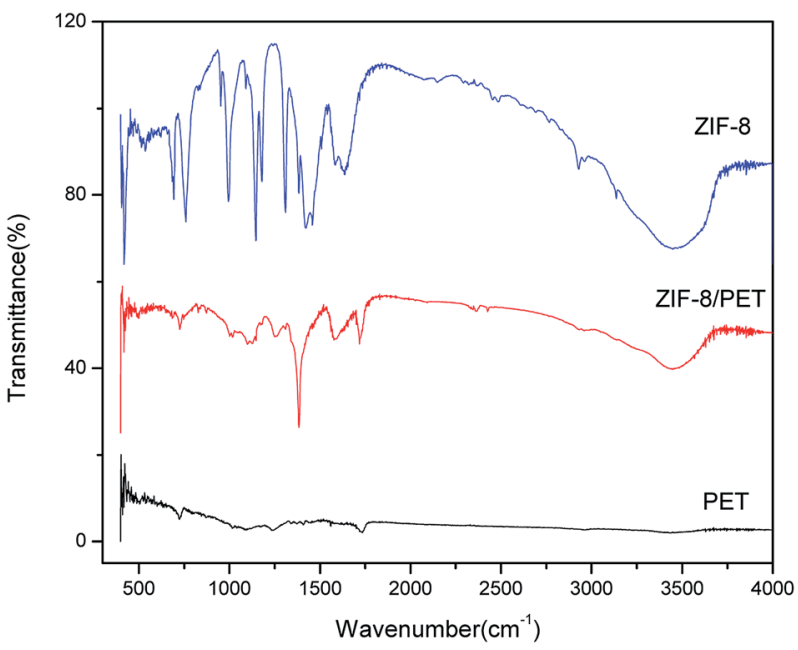

Fig. 6 FT-IR spectra of ZIF-8, PET and ZIF-8/PET. signals at $1300-1460 \mathrm{~cm}^{-1}$ were for the entire ring stretching, whereas band at $1146 \mathrm{~cm}^{-1}$ derived from aromatic $\mathrm{C}-\mathrm{N}$ stretching mode. Similarly, the peaks at 995 and $760 \mathrm{~cm}^{-1}$ could be assigned as $\mathrm{C}-\mathrm{N}$ bending vibration and $\mathrm{C}-\mathrm{H}$ bending mode, respectively. And band at $694 \mathrm{~cm}^{-1}$ was due to the ring out-ofplane bending vibration of the Hmim. Interestingly, the noticeable $\mathrm{Zn}-\mathrm{N}$ stretching vibration band was observed at the position of $426 \mathrm{~cm}^{-1}$, suggesting that zinc ions combined chemically with nitrogen atoms of the methylimidazole groups to form the imidazolate. ${ }^{38}$ Compared with the pure PET, the $\mathrm{N}-\mathrm{H}$ stretching vibration, $\mathrm{C}=\mathrm{C}$ stretch mode, $\mathrm{C}=\mathrm{N}$ stretch vibration, entire ring stretching, aromatic $\mathrm{C}-\mathrm{N}$ stretching mode, $\mathrm{C}-\mathrm{N}$ bending vibration, $\mathrm{C}-\mathrm{H}$ bending mode and $\mathrm{Zn}-\mathrm{N}$ stretching vibration signals appeared in ZIF-8 still could be found in ZIF-8/PET, suggesting the existence of ZIF-8 on the PET. In addition, no new bands appeared in ZIF-8/PET sample. This evidenced that no strong chemical interactions between ZIF-8 particles and PET.

\section{$3.5 \quad \mathbf{P M}_{2.5}$ capture property analysis}

The performances of PET and ZIF-8/PET for $\mathrm{PM}_{2.5}$ capture were tested in a flow-through reactor. As shown in Fig. 7 (a), neat PET and ZIF-8/PET achieved $\mathrm{PM}_{2.5}$ removal efficiency of $38.30 \%$ and $77.15 \%$, respectively. The additions of ZIF-8 made the composites have better performance for $\mathrm{PM}_{2.5}$ removal than pure PET. In addition to removal efficiency, low air resistance is one of the important properties for filters. Both PET and ZIF-8/ PET had a pressure drop of less than $15 \mathrm{~Pa}$. Compared with pure PET, the pressure drop of ZIF-8/PET was higher, which may be attributed to the voids reduction among fiber framework.

Moreover, the recycling performance of ZIF-8/PET was also evaluated. ZIF-8/PET was washed in 50\% methanol after $1 \mathrm{~h}$ $\mathrm{PM}_{2.5}$ capture experiment and then dried in the air. The washed and dried composites were used again to capture $\mathrm{PM}_{2.5}$. It can be seen from Fig. 7(a), the removal efficiency of $\mathrm{PM}_{2.5}$ decreases slightly and remain higher than $74 \%$ after three cycles. The pressure drop increases with the increasing of used times and remain lower than $15 \mathrm{~Pa}$. Xiong et al. ${ }^{39}$ speculated that the residual particles after washed might result in the increase of air resistance, then leading to the increase of pressure drop.

The SEM images of ZIF-8/PET filter before and after $\mathrm{PM}_{2.5}$ removal were exhibited in Fig. 7(b). Tiny particles were captured and attached on the surface of the fibers. The roles of ZIF-8 played in the enhancement of $\mathrm{PM}_{2.5}$ capture property can be concluded as strengthen of adsorption ability. Specifically, the increase of specific surface and micropore volume can provide additional reaction space and adsorption sites. Then, the 


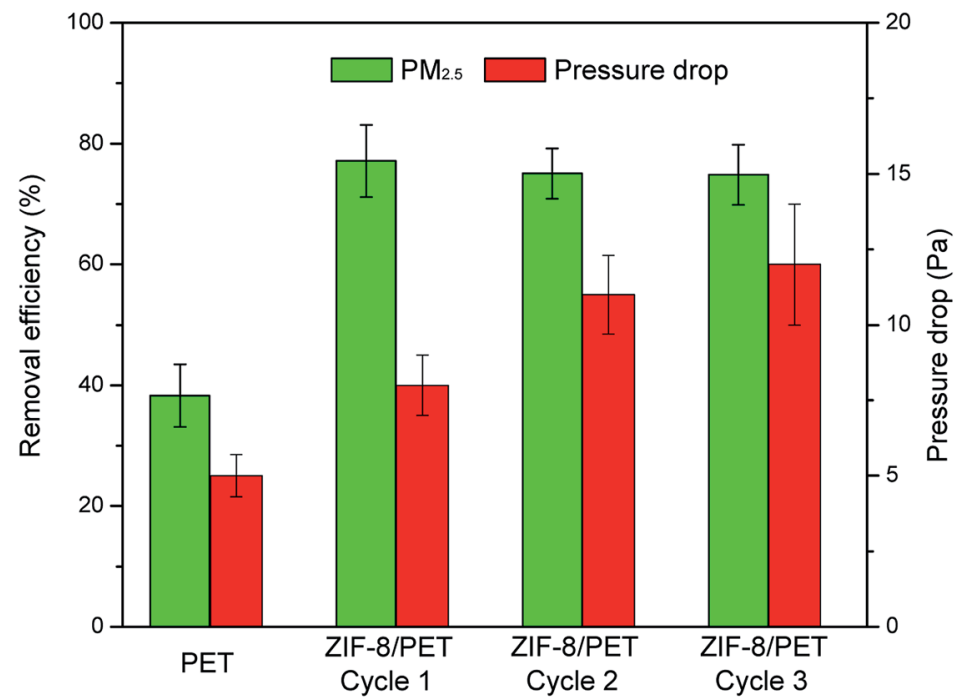

(a)
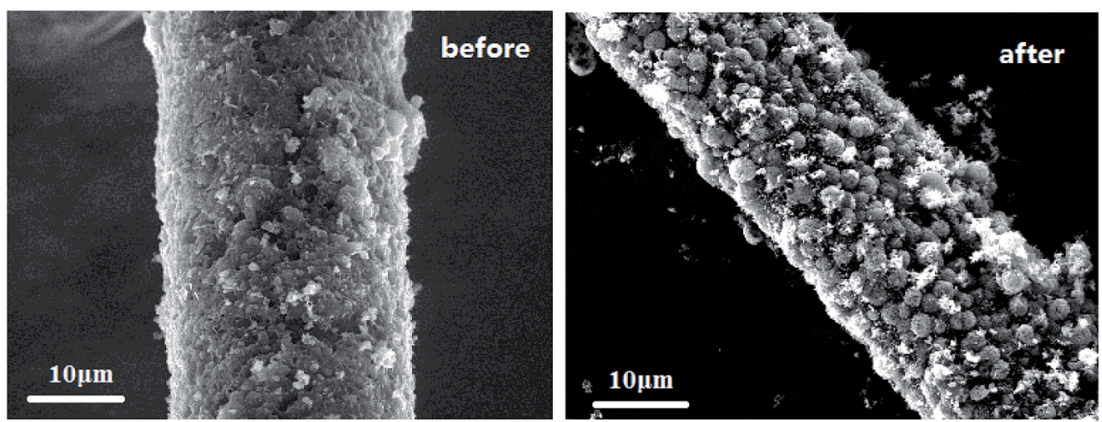

(b)

Fig. 7 (a) $\mathrm{PM}_{2.5}$ removal efficiencies and the pressure drop of PET and ZIF-8/PET. (b) SEM images of the ZIF-8/PET filter before and after PM 2.5 removal.

addition of ZIF-8 enriched the functional groups. Besides, electrostatic interactions between the composites and $\mathrm{PM}_{2.5}$ were another mechanism since ZIF-8 had been reported to exhibit positive surface charges. ${ }^{40,41}$

\section{Conclusion}

ZIF-8/PET was successfully synthesized by growing ZIF-8 on the fibers of non-woven fabric made of PET, which was light and cheap. The ZIF-8/PET material had better activity for $\mathrm{PM}_{2.5}$ capture than neat PET. And the additions of ZIF-8 doubled the filtration efficiency. In addition, the prepared composites showed low pressure drop and good recycling performance. ZIF8/PET was promising for $\mathrm{PM}_{2.5}$ capture due to its unique characters.

\section{Conflicts of interest}

The authors declare no competing interests.

\section{Acknowledgements}

This research was supported by Special Research for PublicBeneficial Environment Protection (No. $\ln 201409080$ ) and the
Key Consulting Project of Chinese Academy of Engineering (No. 2015-06-XZ-01).

\section{References}

1 World Health Organization, http://www.who.int/phe/ health_topics/outdoorair/databases/

FINAL_HAP_AAP_BoD_24March2014.pdf?ua=1, 2014.

2 W. Huang, J. J. Cao, Y. B. Tao, L. Z. Dai, S. E. Lu and B. Hou, Am. J. Epidemiol., 2012, 175(6), 556-566.

3 T. D. Nelin, A. M. Joseph, M. W. Gorr and L. E. Wold, Toxicol. Lett., 2012, 208(3), 293-299.

4 J. Zhou, K. Ito, R. Lall, M. Lippmann and G. Thurston, Environ. Health Perspect., 2011, 119(4), 461-466.

5 R. D. Brook, S. Rajagopalan, C. A. Pope, J. R. Brook, A. Bhatnagar, A. V. Diez-Roux, F. Holguin, Y. Hong, R. V. Luepker and M. A. Mittleman, Circulation, 2010, 121(21), 2331-2378.

6 X. Zhang, Z. Zhao, T. Nordquist and D. Norback, Indoor Air, 2011, 21(6), 462-471.

7 J. G. Watson, J. Air Waste Manage. Assoc., 2002, 52(6), 628713. 
8 V. Ramanathan, P. J. Crutzen, J. Lelieveld, A. P. Mitra, D. Althausen, J. Anderson, M. O. Andreae, W. Cantrell, G. R. Cass, C. E. Chung, A. D. Clarke, J. A. Coakley, W. D. Collins, W. C. Conant, F. Dulac, J. Heintzenberg, A. J. Heymsfield, B. Holben, S. Howell, J. Hudson, A. Jayaraman, J. T. Kiehl, T. N. Krishnamurti, D. Lubin, G. McFarquhar, T. Novakov, J. A. Ogren, I. A. Podgorny, K. Prather, K. Priestley, J. M. Prospero, P. K. Quinn, K. Rajeev, P. Rasch, S. Rupert, R. Sadourny, S. K. Satheesh, G. E. Shaw, P. Sheridan and F. P. J. Valero, J. Geophys. Res., 2001, 106(D22), 28371-28398.

9 C. Wang, S. Wu, M. Jian, J. Xie, L. Xu, X. Yang and Q. Zheng, Nano Res., 2016, 9, 1-8.

10 L. Wang, C. Zhang, F. Gao and G. Pan, RSC Adv., 2016, 6, 105988-105995.

11 S. Jeong, H. Cho, S. Han, P. Won, H. Lee, S. Hong, J. Yeo, J. Kwon and S. H. Ko, Nano Lett., 2017, 17, 4339-4346.

12 H. J. Kim, S. J. Park, C. S. Park, T. H. Le, S. H. Lee, T. H. Ha, H. Kim, J. Kim, C. S. Lee, H. Yoon and O. S. Kwon, Chem. Eng. J., 2018, 339, 204-213.

13 K. S. Park, Z. Ni, A. P. Côté, J. Y. Choi, R. Huang, F. J. UribeRomo, H. K. Chae, M. O'Keeffe and O. M. Yaghi, Proc. Natl. Acad. Sci. U. S. A., 2006, 103(27), 10186-10191.

14 Y. B. He, W. Zhou, G. D. Qian and B. L. Chen, Chem. Soc. Rev., 2014, 43(16), 5657-5678.

15 F. Cacho-Bailo, B. Seoane, C. Tellez and J. Coronas, J. Membr. Sci., 2014, 464, 119-126.

16 P. F. Liu, K. Tao, G. C. Li, M. K. Wu, S. R. Zhu, F. Y. Yi, W. N. Zhao and L. Han, Dalton Trans., 2016, 45(32), 1263212635.

17 A. Jomekian, B. Bazooyar, R. M. Behbahani, T. Mohammadi and A. Kargari, J. Membr. Sci., 2017, 524, 652-662.

18 J. J. Gassensmith, J. Y. Kim, J. M. Holcroft, O. K. Farha, J. F. Stoddart, J. T. Hupp and N. C. Jeong, J. Am. Chem. Soc., 2014, 136(23), 8277-8282.

19 L. Zeng, X. Y. Guo, C. He and C. Y. Duan, ACS Catal., 2016, 6(11), 7935-7947.

20 Z. Tian, X. Yao and Y. Zhu, Microporous Mesoporous Mater., 2017, 237, 160-167.

21 J. Dai, X. Xiao, S. Duan, J. Liu, J. He, J. Lei and L. Wang, Chem. Eng. J., 2018, 331, 64-74.
22 T. T. Bui, N. D. Cuong, Y. S. Kim and H. Chun, Mater. Lett., 2018, 212, 69-72.

23 S. Watanabe, S. Ohsaki, T. Hanafusa, K. Takada, H. Tanaka, K. Mae and M. T. Miyahara, Chem. Eng. J., 2017, 313, 724733.

24 Y. Zhang, S. Yuan, X. Feng, H. Li, J. Zhou and B. Wang, J. Am. Chem. Soc., 2016, 138(18), 5785-5788.

25 Y. Pan, Y. Liu, G. Zeng, L. Zhao and Z. La, Chem. Commun., 2011, 47(7), 2071-2073.

26 A. Schejn, L. Balan, V. Falk, L. Aranda, G. Medjahdi and R. Schneider, CrystEngComm, 2014, 16(21), 4493-4500.

27 J. Font, J. Muntasell and E. Cesari, Mater. Res. Bull., 1999, 34(1), 157-165.

28 R. V. Surendar and A. C. Moises, J. Am. Chem. Soc., 2010, 132(1), 76-78.

29 O. Kolmykov, J.-M. Commenge, H. Alem, E. Girot, K. Mozet, G. Medjahdi and R. Schneider, Mater. Des., 2017, 122, 31-41.

30 L. S. Lai, Y. F. Yeong, N. C. Ani, K. K. Lau and A. M. Shariff, Part. Sci. Technol., 2014, 32(5), 520-528.

31 M. He, J. Yao, Q. Liu, K. Wang, F. Chen and H. Wang, Microporous Mesoporous Mater., 2014, 184, 55-60.

32 D. Liu, Y. Wu, Q. Xia, Z. Li and H. Xi, Adsorption, 2013, 19(1), 25-37.

33 Y. C. Du, J. Z. Sun, S. H. Zhang, R. Q. Hou and H. J. Li, NonMet. Mines, 2016, 39(5), 34-37.

34 J. D. Fairen, S. A. Moggach, M. T. Wharmby, P. A. Wright and S. D. Parsons, J. Am. Chem. Soc., 2011, 133(23), 8900-8902.

35 C. Janosch, M. Simon, L. Sven-Jare, F. Armin, H. Klaus and W. Michael, Chem. Mater., 2009, 21(8), 1410-1412.

36 M. J. C. Ordonez, K. J. Balkus, J. P. Ferraris and I. H. Musselman, J. Membr. Sci., 2010, 361(1-2), 28-37.

37 A. Jomekian, R. M. Behbahani, T. Mohammadi and A. Kargari, Microporous Mesoporous Mater., 2016, 234, 43-54. 38 Y. Hu, H. Kazemian, S. Rohani, Y. Huang and Y. Song, Chem. Commun., 2011, 47(47), 12694-12696.

39 Z. C. Xiong, R. L. Yang, Y. J. Zhu, F. F. Chen and L. Y. Dong, J. Mater. Chem. A, 2017, 5(33), 17482-17491.

40 B. K. Jung, J. W. Jun, Z. Hasan and S. H. Jhung, Chem. Eng. J., 2015, 267, 9-15.

41 K. Y. A. Lin, F. K. Hsu and W. D. Lee, J. Mater. Chem. A, 2015, 3(18), 9480-9490. 